\title{
Bio Remediation of Silver from Waste Using Actinomycetes
}

\author{
Lynn D’ Lima* \& Manju Phadke \\ Department of Microbiology, S.I.E.S College of Science, Arts and Commerce, Sion West, Mumbai-400022
}

\begin{abstract}
Nanotechnology has gained tremendous impetus in the synthesis and development of various metal nanoparticles. Chemical and physical methods have successfully produced pure, well-defined nanoparticles, but these techniques are expensive, energy consuming and potentially toxic to the environment. Therefore, there is a need for environmental friendly approaches for the production of nanoparticles. The aim of this study was to develop a biological process using lesser explored Actinomycete sp. and to convert huge amounts of generated radiographic waste, a potential source for the recovery of valuable silver nanoparticles. Actinomycetes sp. were isolated from different soil samples and were screened for their ability to produce silver nanoparticles (AgNP's). Extracellular synthesis of silver nanoparticles was performed using $1 \mathrm{mM}$ silver nitrate solution. This reaction mixture was monitored for a period of $72 \mathrm{hrs}$. The solution had turned reddish brown in colour, a characteristic feature of silver nanoparticle synthesis. The AgNPs were characterized by UV-Visible spectroscopy with the absorption band at $420 \mathrm{~nm}$ which is a typical plasmon resonance band of silver. The SEM results showed that the nanoparticles were spheric in shape with a size between $40 \mathrm{~nm}$ to $60 \mathrm{~nm}$. These AgNP's proved to have anti algal properties. Radiographic plates were used to prepare AgNP's aided by Streptomyces albolongus and these recycled silver nanoparticles can have wide applications.
\end{abstract}

Keywords: Actinomycetes sp., Anti algal, Bio remediation, Silver nanoparticles, Streptomyces sp.

\section{Introduction}

Nanotechnology has been recently established as a new interdisciplinary science. It is mainly concerned with the synthesis of nanomaterials at the atomic level to gain distinctive properties which can be rightfully manipulated for the preferred application. The term 'nano' indicates one billionth or $10^{-9}$ units. It is widely agreed that nanoparticles (NP) are clusters of atoms in the size range of 1-100 nm. It is understood that the properties of a metal NP are determined by its size, shape, composition and structure (Sun, Y.G., 2002).

Nanotechnology has gained tremendous impetus in synthesis and development of nanoparticles such as copper, zinc, titanium, magnesium, gold and alginate with their wide applications in various fields. Nanoparticles, specially silver nanoparticles (AgNPs) are being viewed as fundamental building blocks of nanotechnology, as silver being a good antibacterial agent is effective against antibiotic-resistant bacteria and hence can be used in silver-based antiseptics. Since it is a non toxic natural inorganic metal and possesses low toxicity towards mammalian tissues, it appears as an interesting material to be used in various applications, such as sensor-technology, biological labelling, in optical devices, electronics, as a bactericidal, in cancer treatment and many more biological, medical, and hygienic applications (Singh, D., 2014).

Chemical and physical methods may successfully produce pure, well-defined nanoparticles, but these techniques are more expensive, energy consuming and potentially toxic to the environment (Okafor, F., 2013). Previous studies have shown that chemically produced zerovalent silver nanoparticles (nAg0) were unstable in solution and would easily aggregate with average particle sizes of $<40 \mathrm{~nm}$ or at high concentrations. As a consequence, the specific surface of the nanomaterial decreases (Gusseme, D. B., 2010). Therefore, there is a need for environmentally friendly approaches for the production of nanoparticles. Such demands can be met with biological processes that have been developed using microorganisms.

Biosynthetic methods can employ either microorganism cells such as algae, bacteria, actinomycetes and fungi or plant extracts for nanoparticles production (Okafor, F., 2013).

Actinomycetes are abundantly found in soil. They are micro-organisms that share important characteristics of fungi and prokaryotes such as bacteria. Though researchers have identified a number of bacterial and fungal strains producing nanoparticles, limited research has been done to explore the ability of actinomycetes to produce nanoparticles. Only a few strains of actinomycetes, producing nanoparticles have been reported. Actinomycetes are physiologically very diverse and produce numerous extracellular enzymes and thousands of secondary metabolites as compared to other microorganisms. The focus on actinomycetes has primarily centred on their phenomenal ability to produce secondary metabolites such as antibiotics and bioactive compounds that affect microbial growth. Actinomycetes in general and Streptomyces in particular have the capacity to produce commercially significant bioactive molecules. More recently, they have been found to be a promising source of wide range of important enzymes and also metallic nanoparticles, especially gold and silver. 
Silver is one of the precious and noble metals. It is used in large quantities for many purposes, particularly in the photographic industry due to its photosensitive properties. It has been reported that $25 \%$ of the world's silver needs are supplied by recycling and that $75 \%$ is obtained from photographic waste. In terms of the environment and economy, it is important that silver be recovered from photographic waste. Compared to other films, waste X-ray photographic films contain an appreciable amount of silver. This can be used to prepare silver nanoparticles and these can be used for various applications (Aktas, M. H., 2010).

\section{Materials And Methods}

1. Sample collection: Soil samples were collected from various locations in Mumbai. The sample was sieved and dried separately at $45^{\circ} \mathrm{C}$ for $1 \mathrm{~h}$ in a hot air oven and then cooled to room temperature. (Nanjwade., 2010).

2. Isolation of Actinomycetes: A series of sterile dilution tubes containing $9 \mathrm{ml}$ of saline was taken. $1 \mathrm{~g}$ of soil was transferred aseptically to the 1 st tube $\left(10^{-1}\right)$ and mixed well. Further serial dilutions were performed to produce $10^{-7}$ suspensions. Suspension $(0.1 \mathrm{ml})$ from each culture tube was spread on sterile actinomycetes isolation agar (AIA) medium plates containing fluconazole $50 \mu \mathrm{g} / \mathrm{ml}$. The plates were incubated at room temperature for 1 week. The plates were observed intermittently during incubation. After $72 \mathrm{~h}$, pin-point colonies, characteristic of actinomycetes were observed and further purified. The isolates were streaked on the media surface aseptically and incubated at room temperature for 7 days. Morphological properties such as colony characteristics, type of areal hyphae, growth of vegetative hyphae, fragmentation pattern and spore formation were observed. Nanjwade., 2010. The purified isolates were preserved on slants at $4^{\circ} \mathrm{C}$ for two months and at $-20{ }^{\circ} \mathrm{C}$ in the presence of glycerol $(15 \% \mathrm{v} / \mathrm{v})$ for longer periods (Rahman, A. M., 2011). Identification of the isolates was performed using 16s rRNA gene sequencing.

3. Silver nanoparticle production: The actively growing culture in a growth medium, actinomycete isolation medium, was used to determine the extracellular nanosilver production. The biomass and liquid medium were separated using Whatmann's filter paper No $1.50 \mathrm{ml}$ of filtrate was mixed with $50 \mathrm{ml}$ of sterilized $\mathrm{AgNO}_{3}$ solution at a final concentration of $1 \mathrm{mM}$. All the reaction mixtures were incubated at room temperature for a period of 72-120 hours. Controls were used for comparison at all stages. The samples were visually observed periodically after $24,48,72$ and 120 hours to check for the nanoparticle formation (Das, V. L., 2014).

4. Study of Stationary Vs Static conditions: In this study, two sets of two flasks were inoculated with the culture. One set was incubated on shaker conditions and the other was kept stationary for a period of 10 days. $50 \mathrm{ml}$ of filtrate was mixed with $50 \mathrm{ml}$ of sterilized $\mathrm{AgNO}_{3}$ solution at a final concentration of $1 \mathrm{mM}$. All the reaction mixtures were incubated at room temperature for a period of 72-120 hours. Controls were used for comparison at all stages. The samples were visually observed periodically after 24, 48, 72 and 120 hours to check for the nanoparticle formation (Das, V. L., 2014).

5. Characterization of synthesized silver nanoparticles. The characterization of the nanoparticles was performed by U.V visible spectrophotometer between $350 \mathrm{~nm}$ to $500 \mathrm{~nm}$.

6. Determination of nitrate reductase activity: The organism was tested for the presence of nitrate reductase enzyme, the probable reducing agent for silver nanoparticle formation (Naveen, K .S. H, 2010; Rahman, A. M., 2011).

7. Determination of Anti-Algal Property: $50 \mathrm{ml}$ of Algae culture medium was inoculated with $50 \mathrm{ml}$ of pond water containing algae in a flask, obtained from Sion Talao, Mumbai. $5 \mathrm{ml}$ and $10 \mathrm{ml}$ of the synthesized nanosilver solutions were added to different inoculated algae culture medium flasks. Control was maintained without the addition of any nanoparticle solution. The flasks were incubated for 7 days and samples were later viewed microscopically under magnification $45 \mathrm{X}$.

8. Silver nanoparticle production from waste radiographic films: The waste radiographic films were cut manually into pieces with scissors and the procedure by Aktas et al, 2010 was followed.

\section{Results and Discussion}

Screening of actinomycetes for nanosilver production: Thirty nine isolates were isolated from various soil samples. The colonies were mostly pinpoint, filamentous and sporulating in nature. Out of the various isolates, the isolate showing the best results was used to conduct the study. Silver nanoparticles were synthesized using the isolated actinomycete strain. The isolate obtained was off white in colour with a rough powdery appearance. The culture was identified as Streptomyces albolongus using 16s rRNA bacterial gene sequencing. After growing the culture for a period of 10 days, the filtrate was found to have produced silver nanoparticle. During a period of $72 \mathrm{hrs}$, the solution changed from pale yellow to a reddish brown colour indicating a reduction process to form silver nanoparticles (Fig 1). This appearance of reddish brown and a peak observed at wavelength $420 \mathrm{~nm}$ under UV visible spectrophotometer (Fig 2) is a clear indication of the reduction of silver ions to give rise to nanosilver, produced extracellularly. There were no other peaks located 
around 350 and $500 \mathrm{~nm}$, indicating the absence of nanoparticle aggregation. Broadening of peaks indicate that the particles are polydispersed (Jain D., 2009). Also sedimentation was not observed further indicating its stability.

The study of growth under aeration conditions i.e. shaker vs stationary showed interesting results. Studies show that micro organisms usually prefer aeration for optimum growth.

In the case of actinomycete species isolated, luxuriant growth was observed in both cases. However, stationary condition was most suitable for extracellular nanoparticle synthesis. Shaker conditions did not show the characteristic colour change to reddish brown even after $72 \mathrm{hrs}$. Thus, indicating that the production of the causative reducing agent may be affected under aeration conditions.

The isolate was found positive for the nitrate reductase enzyme test (Fig 4), a probable reducing agent for silver nanoparticle formation according to Naveen et al., 2010.

Anti-algal activity: The anti algal properties of AgNP's were assessed after a period of 7 days. The control samples were observed to be green indicating a healthy growth of algae. The flasks containing $5 \mathrm{ml}$ and $10 \mathrm{ml}$ of AgNP's showed a brownish tinge with lack of any green colour characteristic to algae due to chlorophyll. The algal matt was observed to be shredded and emitted a foul smell similar to decomposition. The control was observed with the two samples. The control sample showed healthy green cells of algae. The sample containing $5 \mathrm{ml}$ AgNP's showed shrunken but intact cells with a faint green colour. On the other hand, sample with $10 \mathrm{ml}$ AgNP's showed lysed cells and no green pigmentation (Fig 5). The results have shown that AgNP's can be potential anti algal agents. These results are similar to the study conducted by Phadke et al., 2012.

Silver nanoparticle synthesis using $\mathrm{X}$ ray films: This experiment was an attempt to recycle silver and to prepare nanoparticles. The treatment of $\mathrm{X}$ ray films with $1 \mathrm{M}$ nitric acid was to produce aqueous $\mathrm{AgNO}_{3}$. Aqueous $\mathrm{AgNO}_{3}$ was used as a substrate for extracellular synthesis of nanosilver. The reaction mixture within 24-48 hrs showed colour change and showed a peak at $390 \mathrm{~nm}$ after U.V visible spectrophotometer analysis (Fig 6). The study conducted by Njagi et al., 2011, with sorghum bran extracts, showed a surface plasmon resonance peak centered at a wavelength of $390 \mathrm{~nm}$. This indicates that there could be a possibility of converting waste radiographic plates into valuable silver nanoparticles which can have many applications in the fields of health care and environment.

Morphology of the silver nanoparticles: A CRYO-FEG SEM study was performed for the shape and size studies. The silver nanoparticles were found to be spherical in shape and were between $40 \mathrm{~nm}$ to $60 \mathrm{~nm}$ in size. (Fig7)

\section{Conclusion}

The present study has demonstrated a simple biotechnological process for extracellular synthesis of silver nanoparticles using actinomycetes. The formation of nanoparticles using different medium was investigated and Actinomycete Isolation medium with yeast extract under stationary conditions had the ability to maximize the synthesis of silver nanoparticles. The characterization of the silver nanoparticles was confirmed by UV visible spectrophotometer showing a peak at the wavelength $420 \mathrm{~nm}$. Presence of nitrate reductase activity was confirmed proving to have contributed to nanoparticle formation. These biosynthesized nanosilver was studied to have anti algal activity which could have immense industrial applications. Our study also helps in bio remediation of silver and further reuse of these biosynthesized nanosilver for various environmental applications. This study thus proves to be economical due to extracellular synthesis for easier downstream processing and bio remediation of silver and formation of silver nanoparticles. Further investigation needs to be done to understand and identify the crystallinity and the state of the synthesized silver nanoparticles from waste as well as to determine their stability.

\section{Acknowledgements}

We would like to express our gratitude towards Yaahz Xenomics Coimbatore, Tamil Nadu, for performing the 16s rRNA gene sequencing and IITB, Powai, Mumbai, for performing the CRYO- FEG SEM studies.

\section{Bibliography}

[1]. Aktas, M. H. Morcali and Yucel O., 2010, 'Silver Recovery from Waste Radiographic Films by Cementation and Reduction" .Canadian Metallurgical Quarterly, 49, (2):147-154.

[2]. Chaudhary, S. H., Yadav, J., Shrivastava, R. A., Singh, S., Singh, K. A., Gopalan, N., 2013, “Antibacterial activity of actinomycetes isolated from different soil samples of Sheopur (A city of central India)". Journal of Advanced Pharmaceutical Technology \& Research, 4(2): 118-123.

[3]. Das, V. L., Thomas, R., Varghese, T. R., Soniya, E. V., Mathew, J., Radhakrishnan, E. K., 2014, "Extracellular synthesis of silver nanoparticles by the Bacillus strain CS 11 isolated from industrialized area". Biotech, 4:121-126.

[4]. Gusseme, D. B., Sintubin, L., Baert, L., Thibo, E., Hennebel, T., Vermeulen, G., Uyttendaele, M., Verstraete, W., and Boon, N., 2010, "Biogenic Silver for Disinfection of Water Contaminated with Viruses". Applied and Environmental Microbiology, 76(4):1082-1087.

[5]. Jain D, Kumar D. H., Kachhwaha S., Kothari S. L., 2009, "Synthesis of plant-mediated silver nanoparticles using papaya fruit extract and evaluation of their anti microbial activities". Digest Journal of Nanomaterials and Biostructures, 4:557-563. 
[6]. Nanjwade, K. B., Chandrashekhara S., Prakash S., Goudanavar, Ali M Shamarez and Fakirappa V. M., 2010, . "Production of Antibiotics from Soil-Isolated Actinomycetes and Evaluation of their Antimicrobial Activities". Tropical Journal of Pharmaceutical Research. 9 (4): 373-377.

[7]. Naveen, K .S. H, Kumar, G., Karthik, L., Rao K.V. B., 2010, "Extracellular biosynthesis of silver nanoparticles using the filamentous fungus Penicillium sp". Archives of Applied Science Research, 2 (6): 161-167.

[8]. Njagi C. E., Hui H., Stafford L., Genuino H., Galindo M. H., Collins B. J., Hoag E. G., and Suib L.S., 2011, "Biosynthesis of Iron and Silver Nanoparticles at Room Temperature Using Aqueous Sorghum Bran Extracts”. Langmuir, 27 (1):264-271.

[9]. 9. Okafor, F., Janen, A., Kukhtareva, T., Edwards, V., and Curley, M., 2013, "Green Synthesis of Silver Nanoparticles, Their Characterization, Application and Antibacterial Activity". International Journal of Environmental Research and Public Health, 10: 5221-5238.

[10]. Phadke M. and Patel J., 2012, "Environmental applications of biologically synthesized silver nanoparticles obtained from silver resistant Ps. aeruginosa form cactus rhizosphere". Bionano Fronteir Eco Revolution Sri Lanka: 211-215.

[11]. Rahman, A. M., Islam, Z .M. and U1 Islam, A. M., 2011, “Antibacterial Activities of Actinomycete Isolates Collected from Soils of Rajshahi, Bangladesh", Biotechnology Research International, 1-6.

[12]. Singh, D., Rathod, V., Labeenah, F., Kausar, A., Vidyashree, Nishat, A., Priyanka. B., 2014, "Biologically Reduced Silver Nanoparticles from Streptomyces sp. VDP-5 and its Antibacterial Efficacy”. International Journal of Pharmacy and Pharmaceutical Science Research, 4(2): 31-36.

[13]. Sun, Y.G., and Xia, Y.N., 2002, "Shape-controlled synthesis of gold and silver nanoparticles". Science, $298(5601): 2176$ - 2179.

[14]. Waghmare, S. S., Deshmukh, M. M., and Sadowski, Z., 2014, "Biosynthesis, optimization, purification and characterization of gold nanoparticles". African Journal of Microbiology Research, 8(2): 138-146.

Figure 1: Extracellular synthesis of silver nanoparticles using actinomycetes after 48hrs.

Control(left) and Test (right)

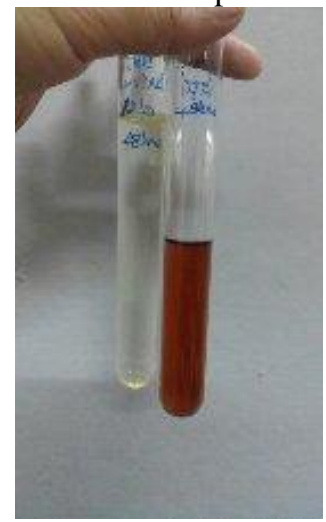

Figure 2: Characterization of biosynthesized nanosilver with U.V visible spectrophotometer.

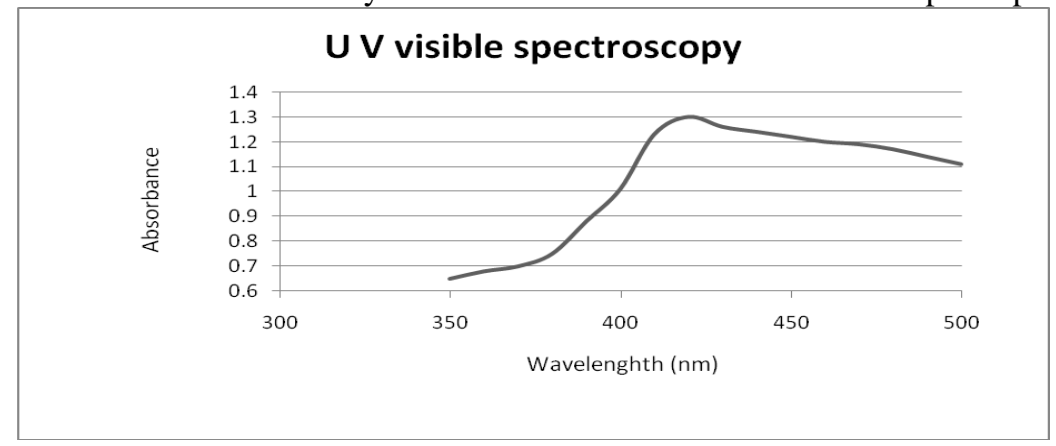

Figure 4: A positive nitrate reductase test showing red colour

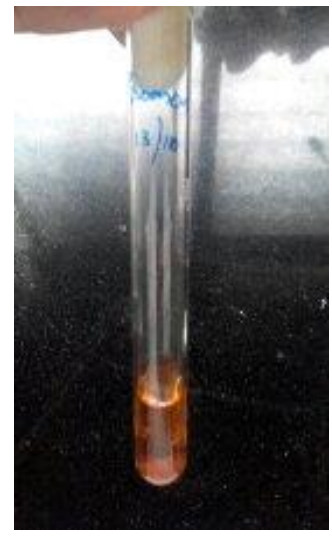


Figure 5: Microscopic images of anti algal activity of nanosilver at 40X



Fig 5.a Control sample with green intact algae cells



Fig 5.b Test (5 ml AgNP's) with shrunken pale green intact algae cells

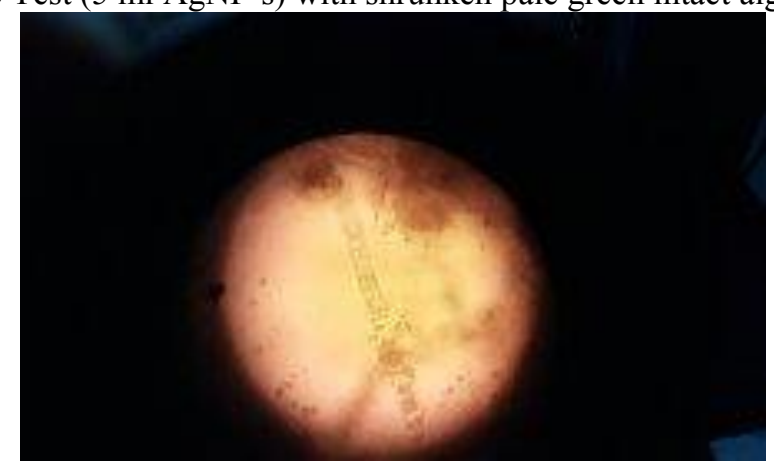

Fig 5.c Test (10 ml AgNP's) with shrunken and lysed algae cells

Figure 6: U.V visible spectroscopy of silver nanoparticle synthesis using X ray films

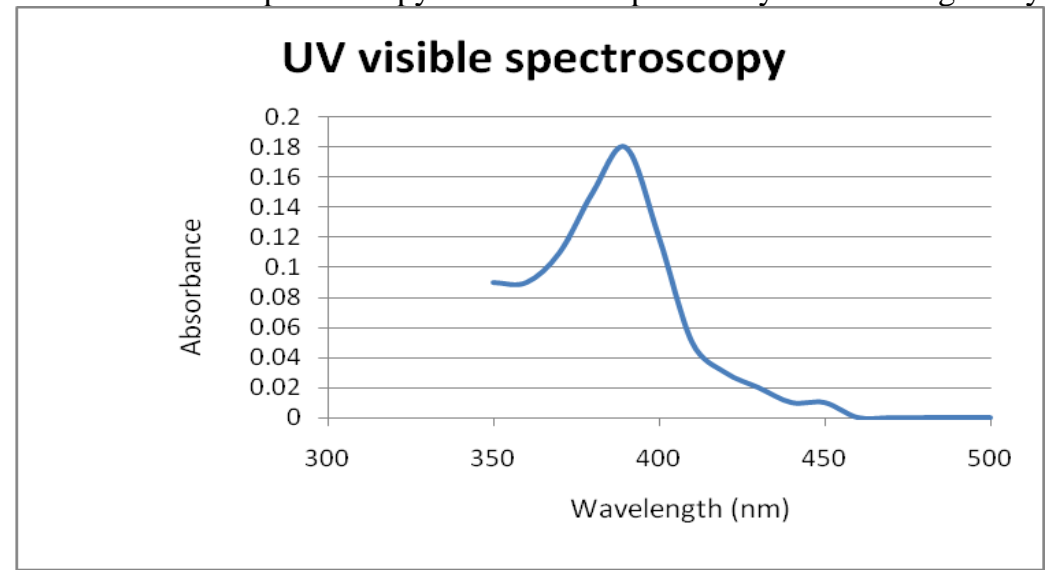


Figure 7: SEM micrograph of AgNPs prepared from the isolate



